\title{
RELACIONES PALEOFLORISTICAS DEL MIOCENO-PLIOCENO DEL NORTE ARGENTINO
}

SILVINA SUSANA GARRALLA

LUISA MATILDE ANZÓTEGUI

LILIA RENÉ MAUTINO

UNNE-CECOAL-CONICET, Ruta 5, km 2,5 (3400) Corrientes, Argentina.

Recibido: 11 de Noviembre de 2015 - Aceptado: 29 de Febrero de 2016

Para citar este artículo: Silvina Susana Garralla, Luisa Matilde Anzótegui y Lilia René Mautino (2016). Relaciones paleoflorísticas del Mioceno-Plioceno del norte argentino. Publicación Electrónica de la Asociación Paleontológica Argentina 16 (1): 1-13.

Link a este artículo: http://dx.doi.org/10.5710/PEAPA.29.02.2016.89

DESPLAZARSE HACIA ABAJO PARA ACCEDER AL ARTÍCULO

Asociación Paleontológica Argentina Maipú $6451^{\circ}$ piso, C1006ACG, Buenos Aires República Argentina

Tel/Fax (54-11) 4326-7563 Web: www.apaleontologica.org.ar

Otros artículos en Publicación Electrónica de la APA 15(1):

\section{de la Fuente \& Sterli}

ESTADO DEL CONOCIMIENTO DE LAS TORTUGAS EXTINTAS DEL TERRITORIO ARGENTINO: UNA PERSPECTIVA HISTÓRICA.

\section{Paulina Carabajal}

GUIA PARA EL ESTUDIO DE LA NEUROANATOMÍA DE DINOSAURIOS SAURISCHIA, CON ENFASIS EN FORMAS SUDAMERICANAS.

\section{Pol \& Leardi}

DIVERSITY PATTERNS OF NOTOSUCHIA (CROCODYLIFORMES, MESOEUCROCODYLIA) DURING THE CRETACEOUS OF GONDWANA. 


\title{
RELACIONES PALEOFLORISTICAS DEL MIOCENO-PLIOCENO DEL NORTE ARGENTINO
}

\author{
SILVINA SUSANA GARRALLA, LUISA MATILDE ANZÓTEGUI Y LILIA RENÉ MAUTINO
}

UNNE-CECOAL-CONICET, Ruta 5, km 2,5 (3400) Corrientes, Argentina.silgabe2@yahoo.com.ar; luisaanzotegui@yahoo.com.ar; liliamautino@yahoo.com.ar

\begin{abstract}
Resumen. Los objetivos de este trabajo son relacionar las paleofloras del Mioceno-Plioceno del norte de la Argentina que caracterizan a cada formación y a cada período, comparar las registradas en el noroeste con las del nordeste a fin de establecer sus diferencias o semejanzas e inferir la evolución florística y paleoambiental a través del intervalo estratigráfico considerado. Las formaciones seleccionadas son las de Paraná e Ituzaingó en el nordeste y las de Anta, San José, Chiquimil, Palo Pintado, Aloformación Playa del Zorro, Andalhuala, del Buey y pozo YPF.SE.x-2 Los Horcones en el noroeste. Se reconocieron 430 especies que fueron volcadas como presencia-ausencia en una base de datos en la que se consignó, para cada una de ellas, familia, formación, tipo de fósil, hábito, comunidad vegetal y representante actual más cercano al fósil. Se aplicó el análisis de agrupamiento simultáneo en modo Q para las formaciones y en modo R para las especies, utilizando la medida de Distancia de Sorensen. Las paleofloras analizadas comparten el 15\% del total de las especies relevadas. El análisis integral comparativo permitió señalar una diferenciación paleovegetacional y paleoambiental entre ambas regiones. En el noroeste, desde el Mioceno temprano al Plioceno se registraron estepas graminosas, bosques ribereños y bosques secos con estacionalidad y sabanas herbáceas, es decir una manifiesta alternancia de cambios ambientales y vegetacionales relacionados al clima. En el nordeste, en cambio, prevalecen los bosques ribereños y bosques secos con estacionalidad de moderados cambios ambientales.
\end{abstract}

Palabras clave. Paleovegetación. Paleoambiente. Neógeno. Norte de Argentina.

Abstract . MIOCENE-PLIOCENE PALEOFLORISTIC RELATIONS OF NORTHERN ARGENTINA. The aims of this paper are to explore the relationships among the Miocene-Pliocene palaeofloras of Northern Argentina, characterizing each formation and period of time, to compare those located in the Northwest with the ones from in the Northeast in order to establish their differences or similarities, and to infer the floristic and paleoenvironmental evolution through the stratigraphic interval considered. The selected formations are Paraná and Ituzaingó in the Northeast and Anta, San José, Chiquimil, Palo Pintado, Playa del Zorro Alloformation, Andalhuala, del Buey, and YPF pit. SE.x-2 Los Horcones in the Northwest. A total number of 430 species were identified and compiled as presence-absence in a database, where each of them was categorized into family, formation, type of fossil, habit, plant community and closest extant representative to fossil species. A cluster analysis was conducted based on the resulted matrix, applying a Q mode for formations and R mode for species, using the Sorensen Distance measure. The paleofloras analyzed share $15 \%$ of the identified species. The comprehensive comparative analysis allowed to distinguish a paleovegetational and paleoenvironmental differentiation between both regions. In the Northwest, from early Miocene to Pliocene, grass steppes, riparian forests, and seasonally dry forests and herbaceous savannas were found; this is an alternating manifest in the vegetational and environmental change related to climate. In the Northeast, instead, riparian forests and dry forests with more seasonal environment change prevailed.

Key words. Paleovegetation. Paleoenvironment. Neogene. Northern Argentina.

LOS ESTUDIOS paleoflorísticos realizados hasta la fecha en el norte de la Argentina, para el intervalo estratigráfico Mioceno-Plioceno, brindaron abundante información taxonómica y paleoambiental puntual para cada formación, e inclusive dentro de cada una, para cada localidad. Sobre la base de los antecedentes registrados, los objetivos de este trabajo son los de relacionar las paleofloras que caracterizan a cada Formación y a cada período y comparar las halladas en el noroeste con las del nordeste de Argentina a fin de es- tablecer sus diferencias o semejanzas e inferir la evolución paleovegetacional y paleoambiental a través del intervalo estratigráfico considerado, ampliando de este modo los conocimientos sobre la paleoflora predominante en la provincia Paleofitogeográfica Neotropical de Argentina (Barreda et al., 2007). Las paleofloras registradas señalan el predominio de diferentes paleocomunidades vegetales y paleoambientes según la edad y formación que las contenga, en general, desarrolladas bajo un clima subtropical a tropical húmedo 
con estacionalidad seca (Lutz, 1979, 1981, 1984, 1986, 1987, 1991; Fernández y Bravo, 1985; Herbst et al., 1987, 2000; Anzótegui y Lutz, 1988; Anzótegui, 1990, 2006; Mautino et al., 1997, 2004; Mautino y Anzótegui, 1998, 2000, 2002a,b, 2014; Anzótegui y Cristalli, 2000; Starck y Anzótegui, 2001; Anzótegui y Herbst, 2004; Zucol et al., 2004; Martínez y Lutz, 2005; Anzótegui et al., 2007a,b; Barreda et al., 2007; Lutz y Martínez, 2007; Anzótegui y Aceñolaza, 2008; Franco y Brea 2008; Franco, 2009; Martínez, 2010, 2014; Mautino, 2010, 2011; Anzótegui y Horn, 2011; Galli et al., 2011; Brea et al., 2013; Brea y Franco, 2013; Franco et al., 2013; Horn, 2014; Vergel et al., 2015).

En el noroeste argentino, para el Mioceno tempranomedio en la Formación Anta (Sierras subandinas en la provincia de Salta), la paleocomunidad inferida a través de los estudios de palinomorfos y esporas de hongos es característica de una estepa graminosa xérica con muy escasa representación de selva subtropical (Rivero de Dibi et al., 2001; Quattrocchio et al., 2003); en la Formación San José (Valles Calchaquíes en las provincias de Salta y Tucumán) del Mioceno medio, se distinguen paleocomunidades higrófitas de bosques de ribera e hidrófilas (acuáticas y palustres) que poblaban áreas próximas a los cursos de agua y xerófilas con sabanas arboladas (Anzótegui y Cristalli, 2000; Anzótegui, 2001, 2002, 2006; Anzótegui y Herbst, 2004; Mautino et al., 2004; Mautino, 2010, 2011; Mautino y Anzótegui, 2014; Vergel et al., 2015). En la Formación del Buey (Sierra de Famatina en La Rioja), de edad cercana al Mioceno medio predomina un paisaje abierto con comunidades halófitas (Barreda et al., 2006), y en el extremo noroeste de Santiago del Estero (Ottone et al., 2013) las asociaciones palinológicas provenientes del pozo YPF.SE.x-2 Los Horcones (Mioceno), se habrían depositado en ambientes dulceacuícolas a salobres (relacionados con una ingresión marina). La cubierta vegetal fue de tipo herbácea-arbustiva de extracción predominantemente xérica. Durante el Mioceno tardío, en la Formación Chiquimil (Valles Calchaquíes en las provincias de Tucumán y Catamarca), prevalecen las sabanas con escasos árboles junto a una comunidad palustre e higrófila pobre que indica déficit de agua y/o presencia de cuerpos ácueos someros (Lutz, 1987; Mautino et al., 1997, 2004; Mautino y Anzótegui, 1998, 2000, 2002a,b, 2014; Anzótegui 2004, 2006; Martínez, 2010, 2014; Mautino, 2010,
2011; Garralla et al., 2012;). En la Aloformación Playa del Zorro (Valle del Cajón en la provincia de Catamarca), Anzótegui et al. (2007a) señalan paleocomunidades de ambientes húmedos propias de un paleolago estable con bosques de ribera pobres y sabanas herbáceas con escasos árboles que prosperaban en ambientes más secos. Para el Mioceno tardío-Plioceno en la Formación Palo Pintado en la provincia de Salta, Anzótegui y Cuadrado (1996); Acevedo et al. (1997); Anzótegui (1998, 2006); Acevedo y Anzótegui (2001); Starck y Anzótegui (2001); Barreda et al. (2007); Lutz y Martínez (2007); Anzótegui y Horn (2011); Galli et al. (2011) y Horn (2014), citan el predominio de paleocomunidades vegetales acuáticas, palustres y de bosques de ribera, con escasa representación de elementos xéricos. Por el contrario, durante el Plioceno temprano, en la Formación Andalhuala (provincia de Catamarca), Menéndez (1982), Fernández y Bravo (1985), Lutz (1987) y Anzótegui et al. (2007b) mencionan la presencia de vegetales xerofíticos, herbáceos y arbóreos con escasa representación de bosques de ribera y de paleocomunidades palustres y acuáticas. En el nordeste argentino, para el Mioceno tardío (sensu Pérez, 2013) en las provincias de Corrientes, Entre Ríos y Santa Fé, se registra en la Formación Paraná una heterogeneidad de ambientes entre marinos y continentales; estos últimos corresponden a ambientes palustres, bosques de ribera y xerófilos (Anzótegui, 1975, 1990; Anzótegui y GarraIla, 1986 1987; Zucol et al., 2004; Anzótegui y Aceñolaza, 2008; Franco y Brea, 2008; Brea et al., 2012, 2013). Para el lapso Plioceno-Pleistoceno se cita la Formación Ituzaingó en las provincias de Corrientes y Entre Ríos (Anzótegui, 1975, 1980, 1987; Lutz, 1979, 1981, 1984, 1986, 1991; Caccavari y Anzótegui, 1987; Anzótegui y Lutz, 1988; Acevedo y Anzótegui, 2001; Zucol et al., 2004; Brea y Zucol, 2007; Franco, 2009, 2010, 2012; Franco y Brea, 2010, 2013, 2015; Franco et al., 2013, 2014; Brea y Franco, 2013) y se postula que la paleovegetación señala la existencia de comunidades dulceacuícolas con desarrollo de bosques de ribera y xerófilos.

Es necesario aclarar que en este trabajo se denomina Aloformación Playa del Zorro a la que es reconocida también como Formación El Morterito (Anzótegui et al., 2007a). Bossi et al. (1992) y Vides y Bazán (2004) dividen a la Formación El Morterito en tres secuencias separadas por dis- 
conformidades: Aloformación Peñas Azules (Secuencia I), Aloformación Playa del Zorro (Secuencia II) y Aloformación El Totoral (Secuencia III), por lo tanto se considera que dichos autores diferencian y delimitan los estratos fosilíferos con mayor precisión.

\section{MATERIALES Y MÉTODOS}

Para este estudio se consideraron todas las especies fósiles dadas a conocer a través de comunicaciones científicas, publicaciones y tesis doctorales previamente citadas, realizadas en la región norte de Argentina. Las formaciones involucradas en el mismo son las de Paraná e Ituzaingó en el nordeste y las de Anta, San José, Chiquimil, Palo Pintado, Aloformación Playa del Zorro, Andalhuala, del Buey y pozo YPF.SE.x-2 Los Horcones en el noroeste (Fig. 1). Los tipos de fósiles registrados en las citadas formaciones y perforación corresponden a palinomorfos (polen, esporas y quistes de resistencia), moldes, leños mineralizados, impresiones de hojas y frutos, y cutículas. Todos los datos recabados fueron volcados como presencia-ausencia en una base de datos donde se consignaron para cada taxa la familia a la que pertenecen, las formaciones que los contienen, tipo de fósil, hábito, comunidad vegetal y representante actual más cercano al fósil. A la misma se aplicó el análisis de agrupamiento simultáneo en modo Q para las formaciones y en modo $\mathrm{R}$ para las especies, utilizando el índice de Distancia de Sorensen. Este índice estadístico mide la similitud, disimilitud o distancias entre sitios o muestras con datos aportados a partir de presencia-ausencia (Ro-

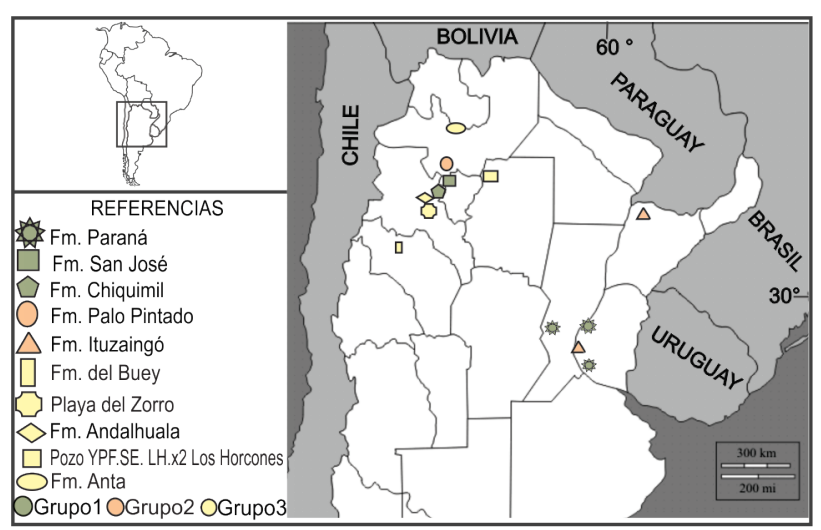

Figura 1. Ubicación geográfica de las formaciones estudiadas. El color de los símbolos de las formaciones responde a los grupos conformados en el análisis de agrupamiento. berts, 1986). La técnica de agrupamiento y gráfico del dendrograma fue realizado mediante el programa PC-ORD (McCune y Mefford, 1999). El dendrograma muestra una escala jerárquica de relaciones según el índice de distancia entre las formaciones y los grupos establecidos (Fig. 2). La confección de la base de datos puso en evidencia una serie de problemas de la paleotaxonomía. Algunas familias actuales contienen granos de polen euripolínicos (gran variabilidad morfológica) que se solapan con otros géneros de la misma o de familias diferentes por tener características semejantes. Esto dificulta la asignación de un taxón fósil a uno actual (familia, género, etc.) ya que tienen afinidad botánica a diferentes grupos de plantas y sus características preservadas resultan insuficientes para una relación taxonómica más estrecha. Por consiguiente en la Tabla 2, en las columnas referidas al afín actual, hábito y comunidad vegetal, se encuentra la palabra incierto (e.g., Chenopodipollis chenopodiaceoides (Martin) Truswell en Truswell, Sluiter y Harris 1985, con características semejantes al complejo Amaranthaceae/Chenopodiaceae que abarca una variedad de ecorregiones).

Para establecer las paleocomunidades se consideraron solo aquellos taxones fósiles cuyos afines actuales poseen distribución acotada y alta especialización ecológica (excepto las formas fósiles de Myrtaceae, tanto polen como hojas, que son semejantes a especies de diferentes tribus, todas de indudable pertenencia a los bosques de ribera. Lo mismo ocurre con los palinomorfos de Poaceae que se los asigna a comunidades xerófilas). Estos casos permiten inferir con cierta probabilidad el tipo de comunidad vegetal que habrían conformado.

Numerosos palinomorfos de las formaciones Paraná e Ituzaingó descriptos en Anzótegui y Garralla (1986) y mencionados en Anzótegui y Lutz (1988) respectivamente, pasaron a sinonimia con géneros fósiles en Mautino (2010), al ser comparados con los de las formaciones San José y Chiquimil. Por otra parte, se asignan frecuentemente distintos nombres genéricos a las partes vegetativas y reproductivas (tallo, hoja, fruto, polen) de una misma planta, o existen diferentes géneros con nomenclatura abierta que pueden ser afines a una misma especie actual. Estas situaciones incrementan el número de especies fósiles presentes en el lapso estudiado y distorsionan el análisis. Ante esta reali- 
dad, para el caso de los nombres fósiles asignados a las distintas partes vegetativas y reproductivas de una misma planta, se los consideró como una sola especie asignándo- les la denominación actual (Tab. 1). Las especies que no están contempladas en esta situación mantienen la denominación original.

TABLA 1. Taxones fósiles según tipo de preservación agrupados por su afín actual.

\begin{tabular}{|c|c|c|c|}
\hline Familia & Taxón fósil & Tipo de preservación & Afin actual \\
\hline \multirow[t]{2}{*}{ Dennstaedtiaceae } & Leptolepidites major Couper 1958 & Polen & Leptolepia spp. \\
\hline & Leptolepidites sp. & & \\
\hline \multirow[t]{2}{*}{ Cyatheaceae } & Cyathidites congoensis Sah 1967 & Polen & Cyathea spp. \\
\hline & $\begin{array}{l}\text { Verrucosisporites subsphaerica Acevedo, Mautino, } \\
\text { Anzótegui y Cuadrado } 1997\end{array}$ & & \\
\hline \multirow[t]{5}{*}{ Anacardiaceae } & Schinus $c f$. terebinthifolius & Impresión & Schinus terebinthifolius \\
\hline & Ailanthipites gamerroi grupo 1 & Polen & \\
\hline & Lithraea $s p$.-Lithraea aff. molleoides & Impresión & Lithraea molleoides \\
\hline & Ailanthipites gamerroi grupo 2 & Polen & \\
\hline & $\begin{array}{l}\text { Astroniumxylon portmanii Brea, Aceñolaza y Zucol } 2001 \text { - } \\
\text { A. bomplandianum Franco } 2009\end{array}$ & Leño & $\begin{array}{l}\text { Astronium urundeuva } \\
\text { (Allemão) Engler } 1881\end{array}$ \\
\hline Celtidaceae & Celtis $s p .1$-Celtis $s p .2$ & Polen & Celtis spinosa - C. pallida -C. tala \\
\hline \multirow[t]{2}{*}{ Euphorbiaceae } & Sapium haematospermoides & Impresión & Sapium haematospermum \\
\hline & Sapium $c f$. haematospermun- Sapium sp. & Polen- Cutícula & \\
\hline \multirow[t]{9}{*}{ Fabaceae } & $\begin{array}{l}\text { Anadenantheroxylon villaurquiense Brea, Aceñolaza } \\
\text { y Zucol } 2001\end{array}$ & Leño & Anadenanthera colubrina \\
\hline & Polyadopollenites $s p$. & Polen & \\
\hline & Prosopis sp. 1 & Impresión & Prosopis sección algarobia \\
\hline & Margocolporites $s p$. & Polen & \\
\hline & Prosopisinoxilon anciborae Martínez 2009 & Leño & \\
\hline & Prosopisinoxylon americanum Franco y Brea 2013 & Leño & Prosopis spp. \\
\hline & Prosocolporites argentina Mautino y Anzótegui 2014 & Polen & \\
\hline & Acaciapollenites myriosporites (Cookson) Mildenhall 1972 & Polen & Acacia spp. \\
\hline & Mimosoxylon sp. & Leño & \\
\hline \multirow[t]{4}{*}{ Lauraceae } & $\begin{array}{l}\text { Lauroxylon artabeae (Brea) Dupéron-Laudoueneix } \\
\text { y Dupéron } 2005\end{array}$ & Leño & Ocotea sp. \\
\hline & Ocotea $s p$ & Cutícula & \\
\hline & Nectandra saltensis Anzótegui & Impresión & Nectandra lanceolata \\
\hline & Nectandra sp. 2 & Cutícula & Nees y Martius 1833 \\
\hline \multirow[t]{3}{*}{ Malvaceae } & Malvacipolloides comodorensis & Polen & Tipo Abutilum pauciflorum \\
\hline & Malvacipolloides sp. & & \\
\hline & Baumanipollis sp. 1 & & \\
\hline \multirow[t]{2}{*}{ Onagraceae } & Corsinipollenites exinitenuis & Polen & Epilobium spp., Ludwigia spp. \\
\hline & Corsinipollenites $s p$. & & \\
\hline \multirow[t]{2}{*}{ Rutaceae } & Rhoipites angurium & Polen & Baulfourodendron riedelianum \\
\hline & Género y especie nueva en leño & Leño & \\
\hline Sapotaceae & Pouteria $s p .1$ - Pouteria sp. 2 & Cutícula & $\begin{array}{l}\text { Pouteria salicifolia (Sprengel) } \\
\text { Radlkofer } 1882\end{array}$ \\
\hline \multirow[t]{2}{*}{ Sparganiaceae } & Sparganiaceaepollenites delicata & Polen & Typha spp. \\
\hline & Sparganiaceaepollenites $s p$. & & Sparganium spp. \\
\hline
\end{tabular}




\section{RESULTADOS}

\section{Análisis de la paleoflora}

Para el Mioceno-Plioceno del norte de la Argentina se relevaron 430 especies fósiles, considerando los argumentos anteriormente discutidos (ver Materiales y Métodos), se redujeron a 407 comprendidas en Chlorophyta, Bryophyta sensu lato y Tracheophyta: Monilophyta, Spermatophyta Gymnospermae y Angiospermae (monocotiledóneas y dicotiledóneas) e Insertae sedis. Los hongos, dinoflagelados y fitolitos no fueron incluidos en este relevamiento. De las numerosas familias que integraron la paleoflora del Neógeno del norte de Argentina, las que presentan mayor diversidad de especies son, en orden de importancia, Cyatheaceae, Lycopodiaceae Osmundaceae, Pteridaceae y Polypodiaceae (Monilophyta), Ephedraceae y Podocarpaceae (Gymnospermae), Anacardiaceae, Arecaeae, Asteraceae, Cyperaceae, Euphorbiaceae, Fabaceae, Malvaceae, Myrtaceae y Poaceae (Angiospermae).

Este análisis pone en evidencia que las paleofloras mio-pliocenas de ambas regiones (nordeste y noroeste del norte argentino) comparten el $15 \%$ del total de las especies fósiles citadas, las que se detallan en la Tabla 2, junto a las formaciones que las contienen, su afín actual, hábito y comunidad vegetal.

Otros aspectos generales que se desprenden de la lectura de la Tabla 2, son: 1) la constante presencia de las especies en las distintas formaciones, lo que señalaría su amplia distribución areal en el lapso analizado. De todas ellas, Podocarpidites marwickii Couper 1953 se destaca por su representación en siete formaciones. Le siguen (solo en cuatro formaciones) Azolla sp., Haloragacidites myriophylloides Cookson y Pike 1954, Haloragacidites sp., Cyperacidites sp.1, Polypodiaceoisporites retirugatus Muller 1968, Plicatella sp., tipo Stryphnodendron, Anadenanthera colubrina (Velloso) Brenan 1955 (por leño y polen), Tubulifloridites antipodica Cookson ex Potonié 1960, Tricolpites trioblatus Mildenhall y Pocknall 1989, Equisetosporites notensis (Cookson) Romero 1977, Equisetosporites claricristatus (Shakmundes) Barreda 1997, Graminidites sp., Araucariacidites australis Cookson 1947, Lithraea molleoides (Velloso) Engler 1876, la mayoría de ellos registrados a partir de palinomorfos y algunos por leños y láminas foliares. El resto de las especies solo son citadas en dos o tres formaciones, indicando su menor distri- bución areal. 2) Desde el Mioceno medio hasta el PliocenoPleistoceno, las especies que señalan una amplia distribución temporal son, en las comunidades acuáticas Azolla sp., Potamogeton sp. y Haloragacidites sp. (Myriophyllum sp.), en el bosque de ribera Cyatheacidites cf. C. annulatus (Lophosoria quadripinnata (J.F. Gmelin) C. Christensen 1920), Polypodiaceoisporites retirugatus Muller 1968 (Pteris denticulata Swartz 1788), Plicatella sp. (Anemia sp.), Ilexpollenites correntina Acevedo y Anzótegui 2001 (/lex sp.), Sapium haematospermum Müller Argoviensis 1865, Anadenanthera colubrina, tipo Stryphnodendron (Stryphnodendron microstachyum Poepp.), Chrysophyllum aff. gonocarpum (Chrysophyllum gonocarpum (Martius y Eichler ex Miquel) Engler 1890) y Syagrus sp. (Syagrus yatay?), en el bosque de altura Podocarpidites marwickii (Podocarpus sp.) y en el xerófilo Lithraea molleoides y Schinopsis lorentzii (Grisebach) Engler 1881. Equisetum sp. (Equisetum giganteum Linnaeus 1759) y Schinus herbstii Anzótegui 1998 (Schinus gracilipes (Johnston 1938)) están citados a partir del Mioceno tardío-Plioceno. Estos aspectos señalan coincidencias con lo expresado por Barreda et al. (2007), en que la paleoflora del norte de Argentina contiene taxones de extracción Neotropical con una distribución más amplia y austral que la actual durante el Mioceno y, particularmente, con la de los actuales Bosques Secos Estacionales Neotropicales (Prado y Gibbs, 1993; Pennington et al., 2000, 2004; Prado, 2000; Franco y Brea, 2008, 2013).

\section{Análisis de agrupamiento}

El análisis de agrupamiento aplicado a todas las formaciones y especies fósiles nos permite diferenciar tres grupos por la medida de distancia entre formaciones (Fig. 2). Las que presentan menor distancia entre ellas por la composición florística similar, son la Formación Paraná con la Formación San José y ambas con la Formación Chiquimil. Otro grupo lo forman la Formación Ituzaingó con la Formación Palo Pintado. Un tercer grupo está integrado por las Formaciones Anta, del Buey, Aloformación Playa del Zorro, Andalhuala y las sedimentitas del pozo YPF.SE.x-2 Los Horcones.

a) Grupo 1. El análisis de agrupamiento de la composición florística de todas las formaciones analizadas indica una mayor similitud entre las formaciones Paraná, San José y 
TABLA 2. Taxones comunes entre formaciones reunidas según el análisis de agrupamiento. Grupo $1 \bigcirc$; Grupo $2 \bigcirc$; Grupo $3 \bigcirc$. 1, Formación Paraná; 2, Formación San José; 3, Formación Chiquimil; 4, Formación Ituzaingó; 5, Formación Palo Pintado; 6, Formación del Buey; 7, pozo YPF. SE.x-2 Los Horcones; 8, Aloformación Playa del Zorro; 9, Formación Andalhuala; 10, Formación Anta.

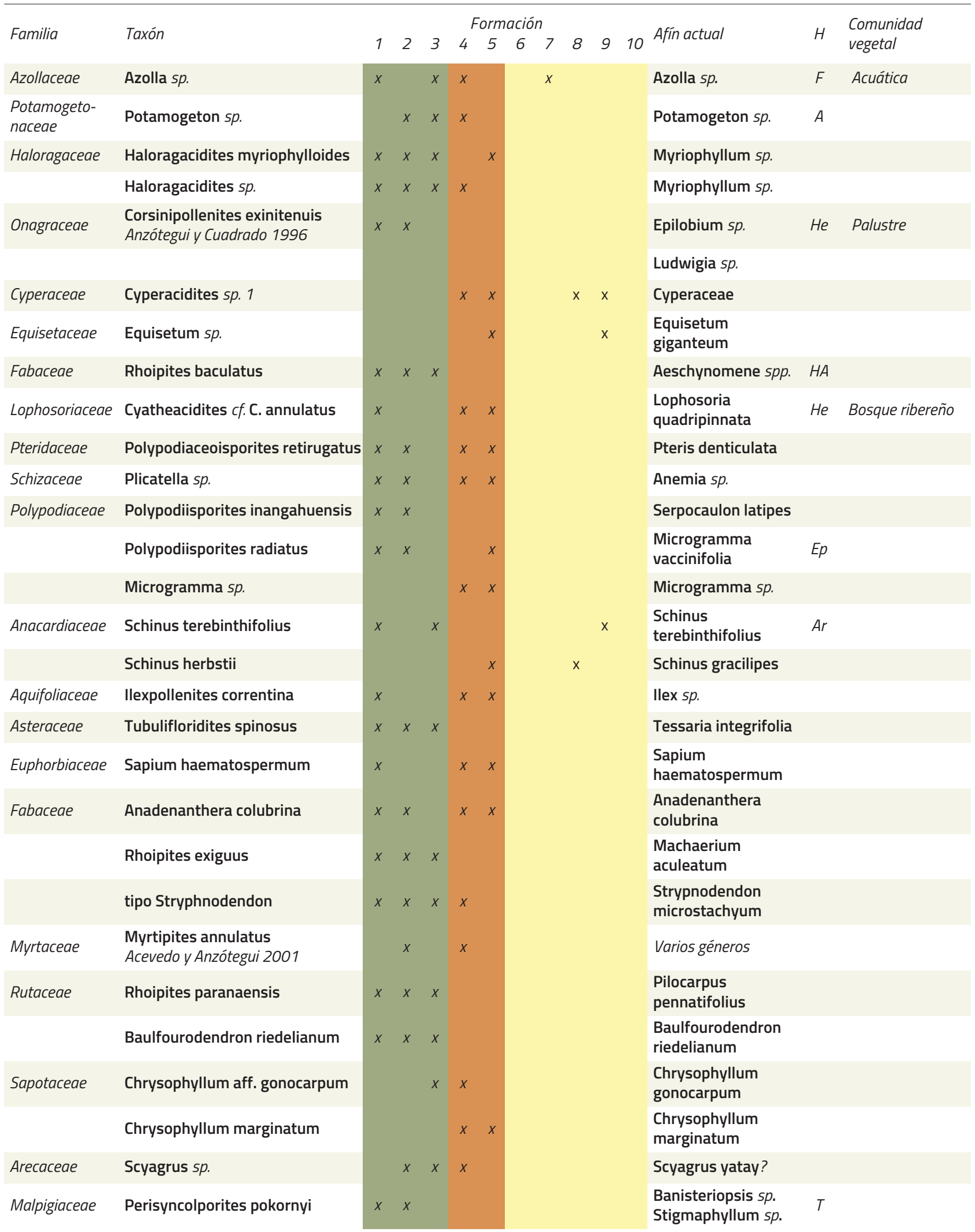




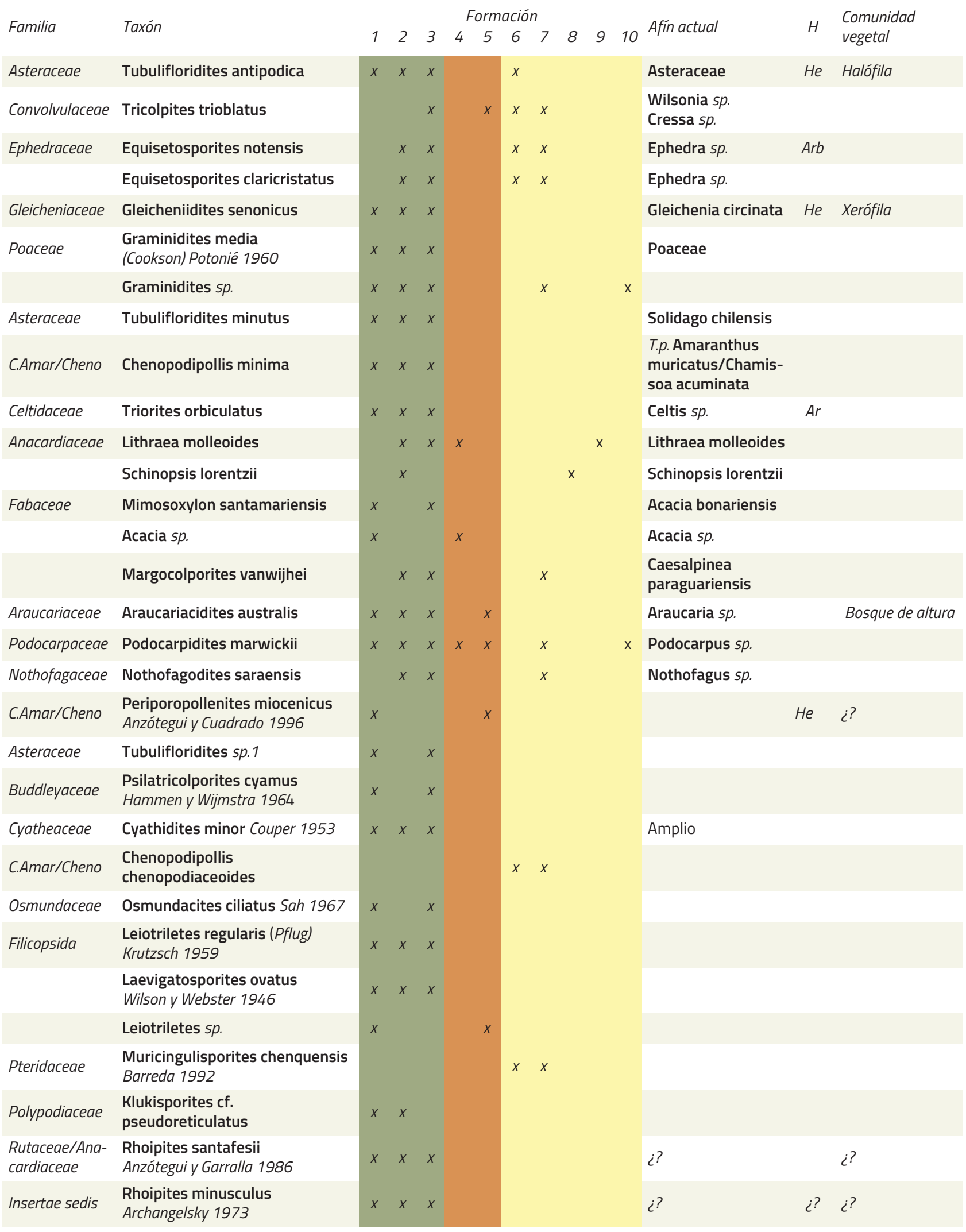

Familias ordenadas de acuerdo a la división vegetal, al hábito y a la comunidad vegetal a la que pertenecen los afines actuales. Abreviaturas: $C$. Amar/Cheno, Complejo Amaranthaceae-Chenopodiaceae; T.p., tipo polínico; $H$, hábito; $F$, flotante; $A$, arraigada; He, herbácea; HA, herbácea-arbustiva; EP, epifita; T, trepadora; Ar, arbóreo; Arb, arbustivo; ¿? incierto. 


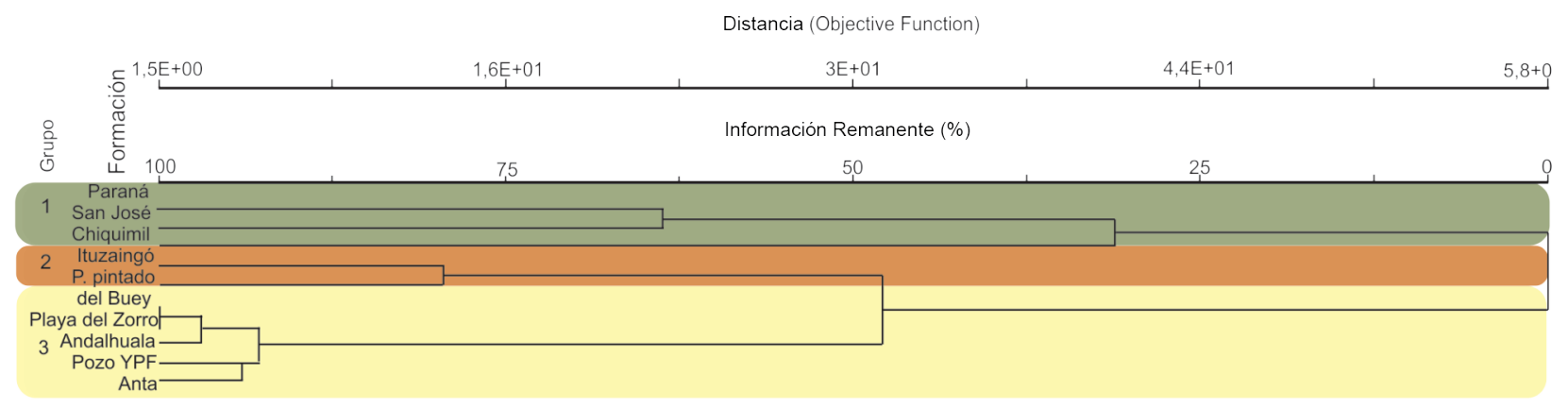

Figura 2. Representación gráfica del agrupamiento entre las formaciones Paraná e Ituzaingó del nordeste de Argentina y San José, Chiquimil, Palo Pintado, Aloformación Playa del Zorro, Anta, del Buey, Andalhuala y Pozo YPF.SE.LH.x-2 Los Horcones del noroeste, utilizando la medida de Distancia de Sorensen.

Chiquimil, del Mioceno medio-tardío, por compartir el $41 \%$ de las especies citadas en la Tabla 2. La mayor cantidad de ellas se concentra entre las que integran las paleocomunidades de bosques de ribera o selva, las xerófilas y las de afinidad incierta. En la primera se encuentran elementos herbáceos y epífitos dados en las Monilophyta: Pteris denticulata (Polypodiaceoisporites retirugatus), Anemia sp. (Plicatella sp.) y Serpocaulon latipes Langsdorff y Fischer 1810 (Polypodiisporites inangahuensis (Couper) Potonié 1956 emend. Pocknall y Mildenhall 1984), la epífita Microgramma vaccinifolia (Langsdorff y Fisch) Copel 1947, (Polypodiisporites radiatus Pocknall y Mildenhall 1984), los arbóreos-arbustivos angiospérmicos, Schinus terebinthifolius Raddi 1820, Tessaria integrifolia Ruiz y Pavón 1798 (Tubulifloridites spinosus Hammen ex Germeraad, Hopping y Muller 1968), Sapium haematospermum, Anadenanthera colubrina, Machaerium aculeatum Raddi 1820 (Rhoipites exiguus Pocknall 1982), Stryphnodendron microstachyum, Pilocarpus pennatifolius Lemaire 1892 (Rhoipites paranaensis Anzótegui y Garralla 1986), Balfourodendron riedelianum (Engler) Engler 1931 y la especie trepadora Banisteriopsis sp. o Stigmaphyllon sp. (Perisyncolporites pokornyi Germeraad, Hopping y Muller 1968). En la comunidad xerofítica se mencionan las herbáceas Gleichenia circinata (Swartz) Cantwell 1967 (Gleicheniidites senonicus Ross 1949), dos especies de Poaceae (Graminidites sp. y G. media (Cookson) Potonié 1960), Solidago chilensis Meyen 1834, (Tubulifloridites minutus (Regali, Uesugui y Santos) Mautino y Anzótegui 2002a), tipo polínico Amaranthus muricatus (Moquin-Tandon) Hieronymus 1882/Chamissoa acuminata Martius 1826 (Chenopodipollis minima Mautino 2011) y cuatro arbóreas o arbustivas, Celtis sp. (Triorites orbiculatus McIntyre 1965), Lithraea molleoides, Acacia bonariensis Gillies ex Hooker y Arnott 1832 (Mimosoxylon santamariensis Lutz 1987) y Acacia sp. El grupo de taxones con afines actuales amplios y comunidad vegetal inciertas (Tab. 2) está integrado en su mayoría por Cyatheaceae, Osmundaceae, Filicopsida indeterminadas y Polypodiaceae, asignables quizá a herbáceas de las comunidades hidrófitas terrestres. Las otras comunidades como las acuáticas, palustres, halofíticas y de bosques de altura cuentan con menos elementos comunes (3-4) entre las tres formaciones, aunque son paleoambientalmente significativos (Tab. 2). Los de la comunidad acuática son Azolla sp., Potamogeton sp. y dos especies de Myriophyllum sp. (Haloragacidites myriophylloides, Haloragacidites sp.); la palustre Epilobium sp. o Ludwigia sp. (Corsinipollenites exinitenuis Anzótegui y Cuadrado 1986), Aeschynomene sp. (Rhoipites baculatus Archangelsky emend. Mautino y Anzótegui 2002). La paleocomunidad halófita está integrada por una Asteraceae (Tubulifloridites antipodica), por Wilsonia sp. o Cressa sp. (Tricolpites trioblatus Mildenhall y Pocknall 1989) y dos especies de Ephedra sp. (Equisetosporites notensis (Cookson) Romero 1977 y E. claricristatus (Shakmundes) Barreda 1997). De los bosques de altura son compartidos solamente Araucaria sp. (Araucariacidites australis Cookson 1947) y Podocarpus sp. (Podocarpidites marwickil).

Por lo tanto, si consideramos el paleoambiente y los elementos comunes entre la Formación San José y Paraná se infiere para la región del norte de Argentina (Anzótegui, 1990, 2006; Anzótegui y Herbst, 2004; Zucol et al., 2004; 
Anzótegui y Aceñolaza, 2008; Franco y Brea, 2008; Mautino, 2010; Brea et al., 2013; Vergel et al., 2015) el predominio durante el Mioceno medio de bosques de ribera sobre los bosque xerófilos, con características ambientales cálidas y más húmedas que en el Mioceno tardío, ya que para esta época la Formación Chiquimil posee elementos vegetacionales de sabanas herbáceas propias de un ambiente cálido y más seco (Mautino, 2010).

b) Grupo 2. Entre las Formaciones Palo Pintado (Mioceno tardío-Plioceno) e Ituzaingó (Plioceno-Pleistoceno) se observa un porcentaje menor de especies en común (14\%), destacándose predominantemente la comunidad de bosque ribereños (higrófilo o selva), constituida en el estrato herbáceo por Lophosoria quadripinnata (Cyatheacidites cf. C. annulatus Cookson 1947), Pteris denticulata (Polypodiaceoisporites retirugatus), Anemia sp. (Plicatella sp.) y la epífita Microgramma sp.; en el arbóreo por llexpollenites correntina (/lex sp.), Anadenanthera colubrina y Chrysophyllum marginatum. También están presentes las Cyperaceae del ambiente palustre y las Podocarpaceae (Podocarpidites marwickii) de los bosques de altura. En consecuencia en el lapso Mioceno tardío-Plioceno-Pleistoceno las citadas formaciones solo tienen en común especies de los bosques de altura y de ribera, pues en la Formación Palo Pintado la vegetación xerofítica es escasa debido a que se desarrolló bajo un clima cálido y más húmedo que en el resto de las formaciones coetáneas (Anzótegui, 1998, 2006; Starck y Anzótegui, 2001; Anzótegui y Horn, 2011; Galli et al., 2011; Horn, 2014). En cambio en la Formación Ituzaingó, principalmente los leños fósiles señalan la presencia de bosques xerófilos e hidrófilos en un clima cálido húmedo estacionalmente seco (Franco, 2009, 2012; Franco y Brea, 2010; Brea y Franco, 2013; Franco y Brea, 2013; Franco et al., 2013, 2014, 2015).

c) Grupo 3. En el grupo formado por las Formaciones Anta (Mioceno temprano), del Buey (Mioceno medio?), Aloformación Playa del Zorro (Mioceno tardío), Formación Andalhuala (Plioceno) y las sedimentitas del pozo YPF.SE.x-2 Los Horcones del Mioceno, comparten escasas especies entre ellas y con las del nordeste. En este grupo se pone de manifiesto el tipo de fósil del cual se extrajeron los datos (palinomorfos, impresiones de hojas), pues en la Formación del Buey y en el pozo YPF.SE.x-2 Los Horcones las especies están representadas por palinomorfos, y en la Aloformación Playa del Zorro y Andalhuala por impresiones de hojas; siendo distintos los requerimientos de fosilización para cada uno de estos fragmentos vegetales.

Las especies compartidas entre la Formación del Buey y las sedimentitas del pozo YPF.SE.x-2 Los Horcones son características de un paisaje abierto herbáceo-arbustivo con comunidades halófitas y xerófitas integradas principalmente por Chenopodiaceae y Convolvulaceae (Wilsonia sp., Cressa sp. (Tricolpites trioblatus)), especies de Ephedra (Equisetosporites notensis y Equisetosporites claricristatus) e indeterminadas (Chenopodipollis chenopodiaceoides y Muricingulisporites chenquensis Barreda 1992). Otros taxones también señalan la presencia de cuerpos de agua dulce semiperennes o salobres; los salobres indicarían una ingresión marina en la región del pozo YPF.SE.x-2 Los Horcones (Ottone et al., 2013) por la presencia de dinoflagelados y foraminíferos. Por otra parte, las ciperáceas de las paleocomunidades palustres, solo son citadas para la Aloformación Playa del Zorro y Formación Andalhuala. La Formación Anta comparte con la mayoría de las formaciones dos especies de palinormofos, Podocarpidites marwickii y Graminidites sp., de amplia distribución. Estas paleoasociaciones del noroeste argentino indican un clima cálido y seco desde el Mioceno temprano al Plioceno.

\section{Resultados bioestratigráficos}

La comparación taxonómica entre la palinoflora de Los Horcones (Mioceno medio?) y la de Formación Chiquimil (Mioceno tardío) (Tab. 2) puso en evidencia que de las 23 especies citadas por Ottone et al. (2013), el 32\% de ellas (Azolla sp., Tricolpites trioblatus (Wilsonia sp., Cressa sp.), Equisetosporites notensis y E. claricristatus (Ephedra sp.), Graminidites sp. (Poaceae), Podocarpidites marwickii (Podocarpus sp.), Margocolporites vanwijhei Germeraad, Hooping y Muller 1968 (Caesalpinea paraguariensis (D. Parodi) Burkart 1952), Nothofagodites saraensis Menéndez y Caccavari 1975 (Nothofagus sp.) es compartido por ambas, si bien estas especies poseen amplia distribución paleogeográfica y cronoestratigráfica para ser señaladas como guías. No obstante, se debe destacar que en la Formación Paraná, datada recientemente como Mioceno tardío (Pérez, 2013) se hallaron dinoflagelados (Anzótegui y Garralla, 1986), entre ellos Spiniferites Mantell, depositados durante la Ingresión Marina 
Paranaense. Si se considera que Ottone et al. (2013) asignan a la palinoflora de Los Horcones, con un margen de dudas al Mioceno medio, por la similitud hallada en este trabajo quizá pueda ser incluida en el Mioceno tardío.

\section{CONCLUSIÓN}

El análisis integral comparativo entre las paleofloras del nordeste y del noroeste, permite señalar que desde el Mioceno temprano y más específicamente desde el Mioceno medio, estas presentaron una marcada diferenciación. Esto se apoya en el hecho de que solo un 15\% del total de especies estaban representadas en ambas regiones, porcentaje que disminuye paulatinamente hacia el Mioceno tardío y Plioceno; si bien en ambas regiones durante el Mioceno las condiciones paleoclimáticas estuvieron vinculadas a un clima cálido subtropical a tropical, con estacionalidad seca. En el Mioceno temprano-medio del noroeste (Tab. 3) se registró un ambiente sumamente árido con escasa vegetación (Formación Anta); en el Mioceno medio se manifestaron dos situaciones, por un lado la continuación del ambiente seco por la presencia de sabanas herbáceas con escasos árboles (Formación del Buey y pozo YPF.SE.X-2 Los Horcones) y por el otro un mejoramiento de las condiciones ambientales por el registro de bosques ribereños y sabanas arboladas (equivalentes a los actuales Bosques secos con estacionalidad) indicadores de mayor humedad (Formación
San José). En el Mioceno tardío para esta misma región, se infiere un desmejoramiento climático (con respecto a la situación anterior) a partir del registro de bosques ribereños acompañados de sabanas herbáceas (Formación Chiquimil y Playa del Zorro). Luego en el Mioceno tardío-Plioceno las condiciones ambientales se tornan localmente más húmedas por el predominio de bosques de ribera, acompañados de pantanos permanentes y escasos representantes xéricos (Formación Palo Pintado). En esta época (Mioceno tardío) en el nordeste se registran bosques ribereños y xerófilos (Formación Paraná) equivalentes, quizá, a los del Mioceno medio. En el Plioceno del noroeste continuó la tendencia a las condiciones xéricas por la presencia de sabanas herbáceas con escasos árboles (Formación Andalhuala). En cambio en el lapso Plioceno-Pleistoceno del nordeste, persistieron los bosques ribereños con predominio de los xerófilos (Formación Ituzaingó), revelando un clima más seco que el de la Formación Paraná pero más húmedo que el de la Formación Andalhuala en el noroeste. Esto pone en evidencia que en el noroeste la paleovegetación fluctuó entre estepas graminosas, bosques ribereños y sabanas herbáceas más secas; mientras que en el nordeste se mantuvieron los bosques con cambios ambientales más moderados. Por lo tanto el análisis de la paleovegetación del norte de Argentina confirma lo ya señalado por otros autores (en Barreda et al., 2007) que en el noroeste, desde el

TABLA 3. Cambios vegetacionales del norte argentino en relación con la edad, Formación y región.

\begin{tabular}{|c|c|c|c|c|}
\hline \multirow[t]{2}{*}{ Edad } & \multicolumn{2}{|c|}{ Región Noroeste } & \multicolumn{2}{|c|}{ Región Nordeste } \\
\hline & Formación & Vegetación & Formación & Vegetación \\
\hline Mioceno temprano-medio & Anta & Estepa graminosa & ¿? & ¿? \\
\hline \multirow[t]{2}{*}{ Mioceno medio } & $\begin{array}{l}\text { del Buey Pozo YPF. } \\
\text { SE.x-2 Los Horcones }\end{array}$ & $\begin{array}{l}\text { Sabana herbácea-arbustiva } \\
\text { xérica }\end{array}$ & ¿? & ¿? \\
\hline & San José & $\begin{array}{l}\text { Bosques ribereños y sabanas } \\
\text { arboladas }\end{array}$ & & \\
\hline Mioceno tardío & $\begin{array}{l}\text { Chiquimil } \\
\text { Playa del zorro }\end{array}$ & $\begin{array}{l}\text { Bosques ribereños y sabanas } \\
\text { herbáceas }\end{array}$ & Paraná & $\begin{array}{l}\text { Bosques ribereños } \\
\text { y bosques xerófilos }\end{array}$ \\
\hline Mioceno tardío-Plioceno & Palo Pintado & $\begin{array}{l}\text { Bosques ribereños, } \\
\text { escasos representantes xéricos }\end{array}$ & & \\
\hline Plioceno & Andalhuala & $\begin{array}{l}\text { Sabanas herbáceo-arbóreas. } \\
\text { Escasos representantes ribereños }\end{array}$ & & \\
\hline Plioceno-Pleistoceno & ¿? & ¿? & Ituzaingó & $\begin{array}{l}\text { Bosques ribereños con } \\
\text { predominio de bosques } \\
\text { xerófilos }\end{array}$ \\
\hline
\end{tabular}


Mioceno temprano se manifiesta mejor la ciclicidad de estos cambios entre desmejoramiento y mejoramiento climático (períodos más secos y húmedos), situación que se refleja también hasta el Holoceno, tanto a escala regional como mundial. Dichos cambios habrían obedecido a diversos factores, los cuales son entre los más significativos, el efecto continentalidad-oceanidad, cambios en los patrones de circulación atmosférica y oceánica, Ingresión Marina Paranaense, inicio de la orogenia andina y el desmejoramiento climático mundial.

\section{AGRADECIMIENTOS}

Nuestro especial agradecimiento a los revisores por las valiosas sugerencias efectuadas. Este trabajo se realizó en el marco de los siguientes proyectos: 1- Floras neógenas del Norte de Argentina: Análisis comparativo, CONICET (PIP 112 20110100417). 2- Paleoasociaciones (invertebrados y flora) del Neógeno del Noroeste de Argentina: Taxonomía, Bioestratigrafía y Paleoambiente, Agencia y UNNE (PICT2011-0216)

\section{REFERENCIAS}

Acevedo, T.L. y Anzótegui, L.M. 2001. Fossil pollen of Myrtaceae from the Upper Tertiary of northern Argentina. Taxonomical Consideration. En: D.K. Goodman, and R.T. Clarke (Eds.). Proceedings of the International Palynological Congress (Houston), AASPF: 311-318.

Acevedo, T.L., Mautino, L.R., Anzótegui, L.M. y Cuadrado, G. 1997. Estudio palinológico de la Formación Palo Pintado (Mioceno superior), Provincia de Salta, Argentina. Parte II: Esporas. Geociências 2: 112-120.

Anzótegui, L.M. 1975. Esporomorfos del Terciario superior de la Provincia de Corrientes (Argentina). $1^{\circ}$ Congreso Argentino de Paleontología y Bioestratigrafía, (Tucumán), Actas 2: 318-329.

Anzótegui, L.M. 1980. Cutículas del Terciario superior de la Provincia de Corrientes, República Argentina. $2^{\circ}$ Congreso Argentino de Paleontología y Bioestratigrafía y $1^{\circ}$ Congreso Latinoamericano de Paleontología (Buenos Aires), Actas 2: 141-167.

Anzótegui, L.M. 1987. Arquitectura foliar de una Lauraceae fósil (Plioceno superior) de Corrientes y su comparación con géneros actuales. $7^{\circ}$ Simposio Argentino de Paleobotánica y Palinología (Buenos Aires), Actas: 111-112.

Anzótegui, L.M. 1990. Estudio palinológico de la Formación Paraná (Mioceno superior) "Pozo Josefina", Provincia de Santa Fé, Argentina. II Parte: Paleocomunidades. Facena 9: 75-86.

Anzótegui, L.M. 1998. Hojas de angiospermas de la Formación Palo Pintado, Mioceno superior, Salta, Argentina. Parte I: Anacardiaceae, Lauraceae y Moraceae. Ameghiniana 35: 25-32.

Anzótegui, L.M. 2001. Bulnesia (Zygophyllaceae) en la Formación San José (Mioceno medio), provincia de Tucumán, Argentina. Boletín de la Sociedad Argentina de Botánica. Suplemento Resúmenes 36: 136.

Anzótegui, L.M. 2002. Hojas de Myrtaceae en la Formación San José (Mioceno medio) Provincia de Tucumán, Argentina. Ameghiniana 39: 321-330.

Anzótegui, L.M. 2004. Megaflora de la Formación Chiquimil, (Mioceno superior), en los valles de Santa María y Villavil, provincias de Catamarca y Tucumán, Argentina. Ameghiniana 41: 303-314.
Anzótegui, L.M. 2006. [Paleofloras del Mioceno en los Valles Calchaquíes, Noroeste de Argentina. Tesis Doctoral, Facultad de Ciencias Exactas y Naturales y Agrimensura, Universidad Nacional del Nordeste, Corrientes, 266 p. Inédita.].

Anzótegui, L.M. y Aceñolaza, P. 2008. Macrofloristic assemblage of the Paraná Formation (Middle-Upper Miocene) in Entre Ríos (Argentina). Neues Jahrbuch für Geologie und Paläontologie-Abhandlungen 248: 159-170.

Anzótegui, L.M. y Cristalli, P. 2000. Hojas de Malvaceae en el Neógeno de Argentina y Brasil. Ameghiniana 37: 169-180.

Anzótegui, L.M. y Cuadrado, G.A. 1996. Palinología de la Formación Palo Pintado, Mioceno superior, Provincia de Salta, República Argentina. Parte I: Taxones nuevos. Revista Española de Micropaleontología 28: 77-92.

Anzótegui, L.M. y Garralla, S.S. 1986. Estudio palinológico de la Formación Paraná (Mioceno superior), (Pozo Josefina), provincia de Santa Fé, Argentina. I parte: Descripciones sistemáticas. Facena 6: 101-177.

Anzótegui, L.M. y Garralla, S.S. 1987. Másulas del género Azolla Lamark, en la Formación Paraná (Mioceno superior), Argentina. $7^{\circ}$ Simposio Argentino de Paleobotánica y Palinología (Buenos Aires), Actas: 113-114.

Anzótegui, L.M., Garralla, S.S. y Herbst, R. 2007. Fabaceae de la Formación El Morterito, (Mioceno superior) del valle del Cajón, provincia de Catamarca, Argentina. Ameghiniana 44: 183-196.

Anzótegui, L.M. y Herbst, R. 2004. Megaflora (hojas y frutos) de la Formación San José (Mioceno medio) en Río Seco, departamento Santa María, provincia de Catamarca, Argentina. Ameghiniana 41: 423-436.

Anzótegui, L.M. y Horn, Y. 2011. Megaflora de la Formación Palo Pintado (Mioceno superior) Salta, Argentina. Parte II. Revista Brasileira de Paleontologia 14: 239-254.

Anzótegui, L.M., Horn, Y.M. y Herbst, R. 2007b. Paleoflora (Fabaceae y Anacardiaceae) de la Formación Andalhuala (Plioceno inferior), provincia de Catamarca, Argentina. Ameghiniana 44: 525-535.

Anzótegui, L.M. y Lutz, A.L. 1988. Paleocomunidades vegetales del Terciario superior (Formación Ituzaingó) de la Mesopotamia Argentina. Revista de la Asociación de Ciencias Naturales del Litoral 18: $105-228$.

Barreda, V.D., Anzótegui, L.M., Prieto, A.R., Aceñolaza, P., Bianchi, M.M., Borromei, A.M., Brea, M., Caccavari, M., Cuadrado, G.A., Garralla, S., Grill, S.G., Guerstein, R., Lutz, A.I., Mancini, M.V., Mautino, L.R., Ottone, E.G., Quattrocchio, M., Romero, E.J., Zamaloa, M.C. y Zucol, A. 2007. Diversificación y cambios de las Angiospermas durante el Neógeno en Argentina. Publicación Especial de la Asociación Paleontológica Argentina 11: 173-191.

Barreda, V.D., Ottone, E.G., Dávila, F.M. y Astini, R.A. 2006. Edad y paleoambiente de la Formación del Buey (Mioceno), sierra de Famatina, La Rioja, Argentina: evidencias sedimentológicas y palinológicas. Ameghiniana 43: 215-226.

Bossi, G.E., Muruaga, C.M., Sanagua, J.G., Hernando, A., Quiroga, G., Ahumada, A.L. y Gavriloff, I. 1992. Aloestratigrafía del Neógeno del valle del Cajón (provincia de Catamarca). $4^{\circ}$ Reunión argentina de sedimentología (La Plata), Actas 3: 137-144.

Brea, M. y Franco, M.J. 2013. El registro fósil de Anacardiaceae en el Cenozoico tardío del Noreste Argentino. Acta Geológica Lilloana 25: 21-36.

Brea, M., Franco, M.J. y Lutz, A.I. 2012. Redescription and reassignment of Entrerrioxylon victoriensis from the Upper Miocene, Paraná Formation, South America. Review of Palaeobotany and Palynology 185: 13-25. 
Brea, M. y Zucol, A.F. 2007. Guadua zuloagae sp. nov., the First Petrified Bamboo Culm Record from the Ituzaingó Formation (Pliocene), Paraná Basin, Argentina. Annals of Botany 100: 711723.

Brea M., Zucol, A.F. y Franco, M.J. 2013. Paleoflora de la Formación Paraná (Mioceno tardío), cuenca chaco-paranaense, Argentina. En: D. Brandoni y J.I. Noriega (Eds.), El Neógeno de la Mesopotamia argentina. Publicación Especial de la Asociación Paleontológica Argentina 14: 28-40.

Caccavari, M. y Anzótegui, L.M. 1987. Polen de Mimosoideae (Leguminosae) de la Formación Ituzaingó, Plioceno superior de Corrientes, Argentina. $4^{\circ}$ Congreso Latinoamericano de Paleontología (Santa Cruz de la Sierra), Actas: 443-458.

Fernández, R.I. y Bravo, E.V. 1985. La presencia del género Acacioxylon (Leguminosae) en sedimentos terciarios del área de los Nacimientos de Abajo (departamento de Belén - provincia de Catamarca) República Argentina. $5^{\circ}$ Simposio Argentino de Paleobotánica y Palinología (Tucumán), Actas: 11.

Franco, M.J. 2009. Leños fósiles de Anacardiaceae en la Formación Ituzaingó (Plioceno-Pleistoceno), Toma Vieja, Paraná, Entre Ríos, Argentina. Ameghiniana 46: 587-604.

Franco, M.J. 2010. Soroceaxylon entrerriensis gen. et sp. nov. (Moraceae) de la Formación Ituzaingó (Plioceno-Pleistoceno), Cuenca del río Paraná, Argentina. Revista Mexicana de Ciencias Geológicas 27: 508-519.

Franco, M.J. 2012. Maderas fósiles de Lauraceae de la Formación Ituzaingó (Plioceno-Pleistoceno), cuenca del río Paraná, Argentina. Revista del Museo Argentino de Ciencias Naturales 14: 307324.

Franco, M.J y Brea, M. 2008. Leños fósiles de la Formación Paraná (Mioceno medio), Toma Vieja, Paraná, Entre Ríos, Argentina: registro de bosques estacionales mixtos. Ameghiniana 45: 699717.

Franco, M.J. y Brea, M. 2010. Microlobiusxylon paranaensis gen. et sp. nov. (Fabaceae, Mimosoideae) from the Pliocene-Pleistocene of Ituzaingó Formation, Paraná Basin, Argentina. Revista Brasileira de Paleontologia 13: 103-114.

Franco, M.J. y Brea, M. 2013. Leños fósiles de leguminosas de la Formación Ituzaingó, Entre Ríos, Argentina: implicancias paleoecológicas y paleobiogeográficas. Ameghiniana 50: 167-191.

Franco, M.J. y Brea, M. 2015. First extra-Patagonian record of Podocarpaceae fossil wood in the Upper Cenozoic (Ituzaingó Formation) of Argentina. New Zealand Journal of Botany 53: 103-116.

Franco, M.J., Brea, M. y Herbst, R. 2014. Palmoxylon romeroisp. nov., de la Formación Chiquimil (Mioceno superior) del Valle de Santa María, Provincia de Catamarca, Argentina. Ameghiniana 51: 572584.

Franco, M.J., Brea, M., Orfeo, O. y Zucol, A.F. 2013. La paleoflora de la Formación Ituzaingó, Argentina. En: D. Brandoni y J.I. Noriega (Eds.) El Neógeno de la Mesopotamia argentina. Publicación Especial de la Asociación Paleontológica Argentina 14: 41-55.

Galli, C., Anzótegui, L.M., Horn, M.Y. y Morton, L.S. 2011. Paleoambiente y Paleocomunidades de la Formación Palo Pintado (Mioceno-Plioceno), provincia de Salta, Argentina. Revista Mexicana de Ciencias Geológicas 28: 161-174.

Garralla, S.S., Morton, L.S., Anzótegui, L.M., Moyano, S. y Herbst, R. 2012. Primeros registros paleontológicos de la localidad de Cerro Pampa (Formación Chiquimil, Mioceno tardío), Provincia de Catamarca, Argentina. GAEA Journal of Geoscience 8: 18-25.

Herbst, R., Anzótegui, L.M., Esteban, G., Mautino, L.R., Morton, S. y Nassif, N. 2000. Síntesis paleontológica del Mioceno de los valles Calchaquíes, noroeste argentino. En: F.G. Aceñolaza y R. Herbst (Eds.). El Neógeno de Argentina, Serie Correlación Geológica, Miscelánea 14: 263-288.

Herbst, R., Anzótegui, L.M. y Jalfin, G. 1987. Estratigrafía, paleoambientes y dos especies de Salvinia Adanson (Filicopsida) del Mioceno superior de Salta, Argentina. Facena 7: 15-42.

Horn, Y. M. 2014. [Palinofloras de las Formaciones El Morterito y Palo Pintado (Mioceno superior-Plioceno inferior), noroeste de Argentina. Tesis Doctoral, Facultad de Ciencias Naturales y Museo de La Plata, Universidad Nacional de La Plata, Buenos Aires, 142 p. Inédita.].

Lutz, A.I. 1979. Maderas de angiospermas (Anacardiaceae y Leguminosae) del Plioceno de la provincia de Entre Ríos, Argentina. Facena 3: 39-63.

Lutz, A.I. 1981. Entrerrioxylon victoriensis nov. gen.et sp. (Leguminosae) del Mioceno Superior (Fm. Paraná) de la provincia de Entre Ríos, Argentina. Facena 4: 21-29.

Lutz, A.I. 1984. Palmoxylon yuqueriense nov. sp. del Plioceno de la Pcia. de Entre Ríos, Argentina. $3^{\circ}$ Congreso Argentino de Paleontología y Bioestratigrafía (Corrientes), Actas: 197-207.

Lutz, A.I. 1986. Descripción morfo-anatómica del estípite de Palmoxylon concordiense Lutz del Plioceno de la Pcia. de Entre Ríos, Argentina. Facena 6: 17-32.

Lutz, A.I. 1987. Estudio anatómico de maderas terciarias del valle de Santa María (Catamarca-Tucumán), Argentina. Facena 7: 125-143.

Lutz, A.I. 1991. Descripción anatómica de Mimosoxylon sp. del Plioceno (Formación Ituzaingó) de la provincia de Corrientes, Argentina. Revista de la Asociación de Ciencias Naturales del Litoral 22: 3-10.

Lutz, A.I. y Martínez, L.C.A. 2007. Nuevo género y especie de liana del Mioceno superior (Formación Palo Pintado) provincia de Salta, Argentina. Ameghiniana 44: 205-213.

Martínez, L.C.A. 2010. Prosopisinoxylon anciborae nov.gen. et sp. (Leguminosae, Mimosoideae) from the Late Miocene Chiquimil Formation (Santa María Group), Catamarca, Argentina. Review of Palaeobotany and Palynology 158: 262-271.

Martínez, L.C.A. 2014. Fossil legume woods from the Late Miocene, Chiquimil Formation (Santa María Basin), Argentina. Review of Palaeobotany and Palynology 201: 1-11.

Martínez, L.C.A. y Lutz A.I. 2005. Primera cita de una leguminosa fósil, Formación Las Arcas, Grupo Santa María, Catamarca, Argentina. Ameghiniana, Suplemento Resúmenes 42: 34R-35R.

Mautino, R.L. 2010. [Palinoflora de las Formaciones San José y Chiquimil (Mioceno medio-superior) Noroeste de Argentina. Tesis Doctoral, Facultad de Ciencias Exactas y Naturales y Agrimensura, Universidad Nacional del Nordeste, Corrientes, 444 p. Inédita.].

Mautino, R.L. 2011. Nuevas especies de palinomorfos de las Formaciones San José y Chiquimil (Mioceno medio y superior), noroeste de Argentina. Revista Brasileira de Paleontologia 14: 279-290.

Mautino, L.R. y Anzótegui, L.M. 1998. Palinología de la Formación Chiquimil, en la localidad Vallecito (Mioceno superior). Parte I: Esporas, Especies nuevas. Ameghiniana 35: 227-233.

Mautino, L.R. y Anzótegui, L.M. 2000. Esporas del Mioceno y retrabajadas del Mesozoico en la Formación Chiquimil, Vallecito, Provincia de Catamarca. Ameghiniana 37: 13-22.

Mautino, L.R. y Anzótegui, L.M. 2002a. Palinología de la Formación Chiquimil (Mioceno superior) Vallecito, provincia de Catamarca. Parte II. Polen. Ameghiniana 39: 257-270.

Mautino, L.R. y Anzótegui, L.M. 2002b. Palinología de la Formación Chiquimil (Mioceno superior), Vallecito, provincia de Catamarca. 
Parte III. Polen. Ameghiniana 39: 271-284.

Mautino, L.R. y Anzótegui, L.M. 2014. Novedades palinológicas en las formaciones San José y Chiquimil (Mioceno medio y superior) noroeste de Argentina. Revista del Museo Argentino de Ciencias Naturales 16: 143-164.

Mautino, R.L., Anzótegui L.M. y Herbst, R. 1997. Análisis palinológico de la Localidad Nacimientos de Abajo, Neógeno, en la Sierra de Hualfin, Departamento Belén, Catamarca, Argentina. Geociencias 2: 121-127.

Mautino, L.R., Cuadrado, A.G. y Anzótegui, L.M. 2004. Novedades taxonómicas, diversidad y significado evolutivo del polen de Malvaceae en el Terciario de Argentina. Revista Española de Micropaleontología 36: 467-483.

McCune, B. y Mefford, M.J. 1999. PC-ORD. Multivariate analysis of Ecological Data, Version 4. MjM Software Desingn, Oregon, 237 p.

Menéndez, C.A. 1982. Leño petrificado de una leguminosa del Terciario de Tiopunco, provincia de Tucumán. Ameghiniana 2: 121-126.

Ottone, E.G., Reinarte Mazurier, S.M.E. y Salinas, A. 2013. Palinomorfos miocenos del subsuelo de Santiago del Estero, Argentina. Ameghiniana 50: 509-521.

Pennington, R.T., Lavin, M., Prado, D.E., Pendry C.A., Pell, S.K. y Butterworth, C.A. 2004. Historical climate change and speciation: neotropical seasonally dry forest plants show patterns of both Tertiary and Quaternary diversification. Philosophical Transactions of the Royal Society of London, Series B 359: 515-358.

Pennington, R.T., Prado, D.E. y Pendry, C.A. 2000. Neotropical seasonally dry forest and Quatenary vegetation changes. Journal of Biogeography 27: 261-273.

Pérez, L.M. 2013. Nuevo aporte al conocimiento de la edad Formación Paraná, Mioceno de la provincia de Entre Ríos. Argentina. En: D. Brandoni y J.I. Noriega (Eds.), El Neógeno de la Mesopotamia Argentina. Publicación Especial de la Asociación Paleontológica Argentina 14: 7-12.

Prado, D.E. 2000. Seasonally dry forest of tropical South America: from forgotten ecosystems to a new hytogeographic unit. Journal of Botany 57: 437-461.

Prado, D.E. y Gibbs, P.E. 1993. Patterns of species distributions in the dry seasonal forests of South America. Annals of Misouri Botanical Garden 80: 902-927.

Quattrocchio, M., Durango de Cabrera, J. y Galli, C. 2003. Formación Anta (Mioceno temprano/medio), subgrupo Metán (Grupo Orán), en el río Piedras, Provincia de Salta. Datos palinológicos. Revista de la Asociación Geológica Argentina 58: 117-127.
Rivero de Dibi, L., Rodríguez de Sarmiento, M. y Durango de Cabrera, J. 2001. Esporas de hongos de la Formación Anta (Grupo Orán) Mioceno medio, provincia de Salta, Argentina. Acta Geológica Lilloana 18: 257-264.

Roberts, D.W. 1986. Ordination on the basis of fuzzy set theory. Vegetation 66: 132-131.

Starck, D. y Anzótegui, L.M. 2001. The late climatic change persistence of a climatic signal through the orogenic stratigraphic record in northwestern of Argentina. Journal South American Earth 14: 763-774.

Vergel, M. del M., Herbst, R., Garralla, S.S., Morton L.S., Anzótegui, L.M., Mautino, L.R., Aráoz, L. y Gavriloff, I.J.C. 2015. Paleontología de invertebrados, icnofósiles, paleobotánica y palinología de Tucumán. En: M.E. Puchulu, L. Ibañez, y G.E. Aceñolaza (Eds.), Geología de Tucumán. $3^{r a}$ Edición. Colegio de Graduados en Ciencias Geológicas de Tucumán, p. 185-207.

Vides, M.E. y Bazán, C.A. 2004. La Aloformación Playa del Zorro, Quebrada del Totoral, Valle del Cajón, provincia de Catamarca. $10^{\circ}$ Reunión Argentina de Sedimentología (San Luis), Resúmenes: 172-174.

Zucol, A.F., Brea, M., Lutz, A. y Anzótegui, L.M. 2004. Aportes al conocimiento de la paleodiversidad del Cenozoico superior del Litoral argentino: Estudios paleoflorísticos. En: F.G. Aceñolaza (Ed.), El Neógeno de Argentina. Serie Correlación Geológica Temas de la Biodiversidad del Litoral Fluvial Argentino, Miscelánea 12: 91-102.

Doi: 10.5710/PEAPA.29.02.2016.89

Recibido: 11 de Noviembre de 2015

Aceptado: 29 de Febrero de 2016 